\title{
An Optical Modulator in Unmodified, Commercially-Available CMOS Technology
}

\author{
Konstantin Nikolic, Alexandru Serb, Student Member, IEEE, and Timothy G. Constandinou, Senior Member, IEEE
}

\begin{abstract}
Here we present a method and structures for mid-infrared, free-space optical communication using unmodified, commercially available complementary metal-oxidesemiconductor integrated circuits. The modulator is based on the free carrier absorption in parasitic PN junction structures under reverse bias. Measured results demonstrate the proofof-concept with speeds of $100 \mathrm{bps}(1.55 \mu \mathrm{m}$ wavelength), but at least two orders of magnitude improvement can be achieved. This technology will enable non-galvanic chip-to-chip and chipto-package communication as an alternative to wirebonding in applications that benefit from a planar top chip surface, such as chemical sensing lab-on-chip systems as well as general sensors and mid-infrared communication.
\end{abstract}

Index Terms-light modulators, CMOS, electro-absorption effect, photonics, silicon

\section{INTRODUCTION}

$\mathbf{M}$ ONOLITHIC integration of optical components and integrated circuits is a key driver in the field of silicon photonics [1], with a strong focus on telecommunication applications. However there is a whole range of possible applications for optical communications which are not in the realm of high-speed telecommunications, but which can benefit from a simple, directly implementable technique which uses light for communication. For example, a serious drawback for many lab-on-chip applications and other hybrid system is the encapsulation of bond wires which drastically reduces the access to the top surface of the chip for any kind of sensors and potential microfluidic system integration. The presence of a simple circuitry on the existing semiconductor chips for outputting information using light, even at moderate speeds, would be very useful. Hence the development of a method for a non-galvanic information output from a semiconductor device or a chip within a low cost integrated technology is therefore of great interest to the industry.

Poor light emitting properties of silicon limit the progress in this area and the ideas for silicon light emitting diodes (LEDs) and lasers thus far [2] were all based on some modifications of complementary metal-oxide-semiconductor (CMOS) fabrication processes, from minimal such as dislocation loops [3] to more significant such as silicon nanocrystals [4], addition of rare-earth materials, Raman effect [5], etc. Another idea of how to output data optically from a CMOS device is to use an external light source and modulate the light in the CMOS

The authors are with the Department of Electrical \& Electronic Engineering and Centre for Bio-Inspired Technology, Institute of Biomedica Engineering, Imperial College London, London SW7 2AZ, UK, email: $\{$ k.nikolic,alexandru.serb05,t.constandinou\}@imperial.ac.uk

The authors greatfully acknowledge the support of the UK EPSRC, grants EP/G070466/1 and EP/H024581/1.

Manuscript received November 30, 2010; revised March 07, 2011.

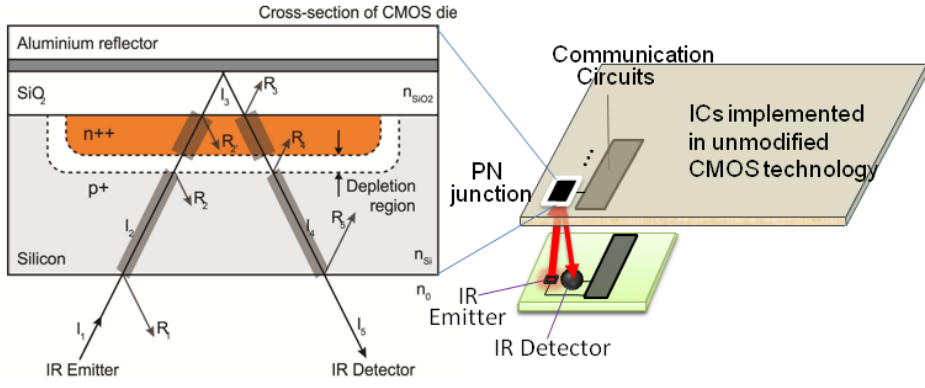

Fig. 1. Principle of operation of a mid-infrared free-space optical communication system. The vertical incidence of the light beam allows two modes of operation: reflective, shown above (similar to the CD/DVD data read), or transmitted beam mode (without the metal reflector). The inset illustrates a crosssection of the communication parasitic PN junction, showing the incident and reflected paths of infra-red radiation through a silicon CMOS die.

structure. The most common optical modulation techniques in silicon are based on the plasma dispersion effect [6] and thus far were usually combined with interference effects, using the bias voltage [7]-[11] or photoexcitation by ultra-fast low energy pump pulses (in all-optical modulators [12]) to generate carriers in Si. Although very successful in achieving very high communication speeds, this approach can be very sensitive to fabrication tolerances and most of the solutions coupled waveguides and separate structures. Treyz et.al [13] reported an all-Si modulator based on free carrier absorption and without use of interference, with a modulation depth of $-6.2 \mathrm{~dB}$ and response time $50 \mathrm{nsec}$. However, this technique is less efficient (carrier injection in [13] requires at least ten times more energy per bit than our solution) and needs dedicated rib waveguides. The vast majority of optoelectronic integrated circuits (ICs) require specialist semiconductor fabrication technologies or additional process steps, which are not yet mainstream. For some applications there is a need to achieve the optical data output using standard, commercially available CMOS ICs.

Here we present a solution to free-space optical communication based on electrically controlled modulation (electroabsorption, due to free carrier absorption) of mid-infrared light sent through free space to a CMOS IC with parasitic PN junction structures, Fig. 1. Both the light emitter and the photodetector are of conventional readily available type and located outside the electronic chip. The proposed technique is not based on interference effects and therefore it is very robust and can be achieved using LEDs and commercial CMOS ICs.

\section{THEORETICAL EXPECTATIONS}

The signal that we measure is the output light intensity $(I)$, either reflected as in Fig. 1 or transmitted as in experiments shown here, which will be attenuated due to free carrier 


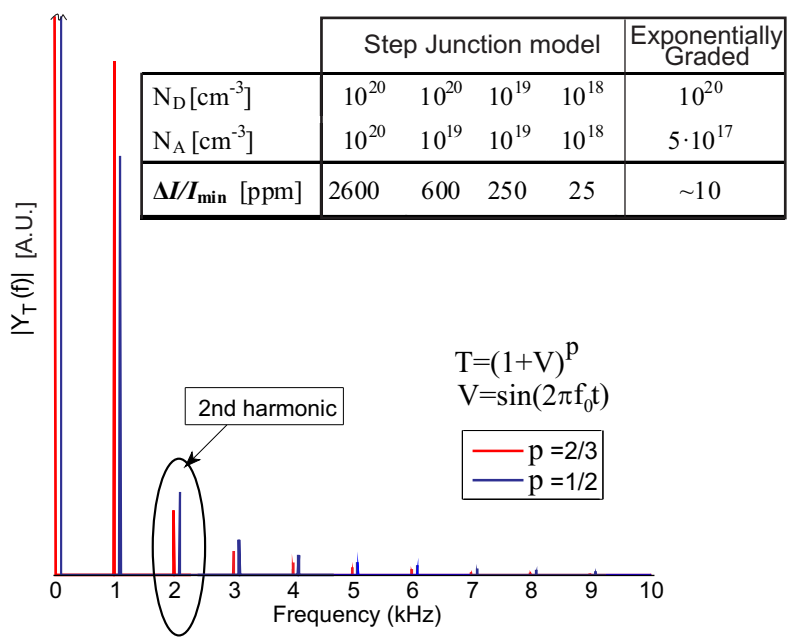

Fig. 2. Table shows theoretical estimates for the maximum modulation due to $\Delta V=5 V$ for the step junction model and for the exponentially graded doping profile assuming $\mathrm{n}+$ diffusion (with surface donor concentration $N_{D}$ ) into a p-well $\left(N_{A}\right)$. Graph shows amplitude spectrums for the depletion region width modulation, i.e. the output signal, for: step junction, $p=1 / 2$ (approximates the well-to-well junction), and an exponentially graded doping profile, $p=2 / 3$ (approximation for diffusion-to-well structures).

absorption. The coefficient of absorption $(\alpha)$ depends on the free carrier concentration, as described by Soref [14], [15] and Lipson [10]. The free carrier concentration can be changed in the region of the $\mathrm{PN}$ junction by changing the applied reverse bias voltage $V$ and thus altering the depletion region width $w$. A detailed analysis of how $w$ and consequently the relative change of light intensity $\delta I=\Delta I / I_{\min }$ and the modulation depth $I_{\max } / I_{\min }$ depend on $V$ are given in our theoretical exposition [16]. Here we summarise the theoretical results in Fig. 2. We assume two types of PN junctions: wellwell and diffusion-into-well, the former was approximated by a step junction and the latter by an exponential doping profile. For a step junction there is a text-book solution [17]: $w \sim \sqrt{V_{\mathrm{bi}}-V}$ where $V_{\mathrm{bi}}$ is the built-in voltage. If $I_{0}$ is the input light intensity, the output at distance $L$ is approximately: $I=I_{0} \exp (-\alpha L)$, what gives $\delta I \sim\left(V_{\mathrm{bi}}-V\right)^{1 / 2}$ (assuming $L=L_{0}-w$ and $\left.\alpha w<<1\right)$. The exponential doping profile requires more calculations and the numerical results [16] show $\delta I \sim\left(V_{\mathrm{bi}}-V\right)^{2 / 3}$. In the experiments we applied a reverse bias to the PN junctions $V=V_{\mathrm{DC}}+V_{0} \sin \left(2 \pi f_{0} t\right)$, hence: $\delta I \sim\left[V_{1}-V_{0} \sin \left(2 \pi f_{0} t\right)\right]^{p}$, where $V_{1}=V_{\mathrm{bi}}-V_{\mathrm{DC}}$. The spectrum of approximate output $\delta I$ is given in Fig. 2. Although the real structures deviate from these perfect cases, the theory indicates the limits and suggests that a very sensitive technique may be needed in order to observe this effect.

\section{Methodology, InstRumentation And RESUlts}

\section{A. CMOS-based optical modulator design. Instrumentation.}

A microphotograph of the test chip together with details of specific optical modulator structures is shown in Fig.3.

The experimental setup describing the individual parts is given in Fig. 4. To demonstrate the modulation we employed the phase-lock technique to detect the signal (after a transimpedance amplifier) using the $2^{\text {nd }}$ harmonic. The $2^{\text {nd }}$ harmonic was specifically targeted to exclude any feedthrough
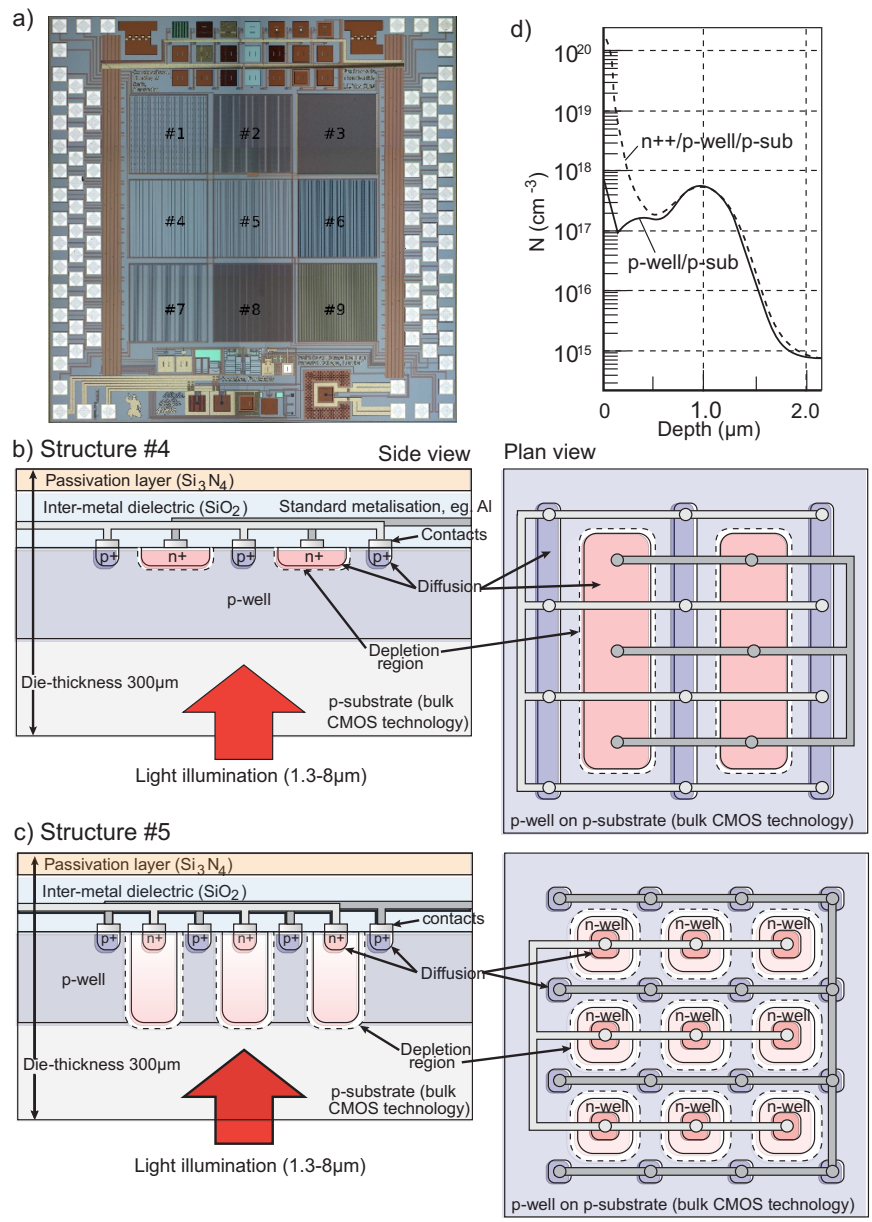

Fig. 3. CMOS Integrated Circuit. (a) Microphotograph of the test chip with nine different $\mathrm{PN}$-junction structures, fabricated in a commercially available $0.35 \mu \mathrm{m}$ CMOS technology. The size of each structure is $500 \times 500 \mu \mathrm{m}$. (b) Test structure \#4: side and plan views of the device consisting of multiple $\mathrm{n}$-diffusion stripes within a single $\mathrm{p}$-well, i.e. $\mathrm{PN}$-junctions are $\mathrm{n}+/ \mathrm{p}$-well. This structure is made up of 12 stripes of n-diffusion of $37 \times 480 \mu \mathrm{m}$. (c) Structure \#5: an array of n-well regions within a p-well/p-substrate, made up of 624 $\mathrm{n}$-wells of size $15 \times 15 \mu \mathrm{m}$ each. (d) Possible doping profile for $\mathrm{n}+\mathrm{p}$-well. The light wavelength $(\lambda)$ is in the range $1.3-8 \mu \mathrm{m}$, where bulk silicon is largely transparent and free-carrier absorption is dominant ( $\alpha \sim \lambda^{2}$, e.g. see [14]).

and it offered the best signal-to-noise ratio in our setup. As the modulation is non-linearly proportional to the applied (reverse) bias, $\delta I(V)$, any signal recovered on the $2^{\text {nd }}$ harmonic is a consequence of the modulation of the free-carrier absorption. The input voltage modulation is in the form of a sine wave of frequency $f$ and a square wave amplitude modulation of $f_{\text {env }}$.

\section{B. Measured Results}

The results demonstrating the achievement of light modulation are shown in Fig. 5. When the light source (LED) is OFF (Fig. 5 top, left of diagram), then the modulation of the depletion width, caused by bias voltage shown in trace 3 (red), has no effect on the output. When light source is ON, after a short transitional period, the bias voltage on the PN junction in the shape of a square wave (modulated sine wave) is faithfully recovered at the output. The result for a well-well structure shows much stronger signal (Fig. 5-bottom), indicating better utilisation of the PN junction area than the junction created by diffusion. The demonstrated speeds are consequence of the 


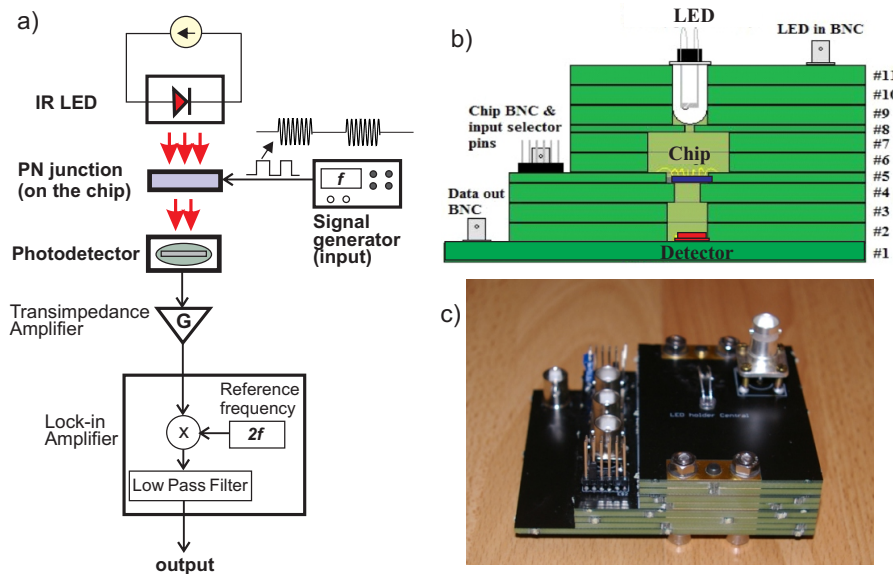

Fig. 4. The experimental setup: (a) Simplified schematic, (b) Cross-section of the printed circuit board (PCB) stack. Nine different PCBs \#8 were fabricated, each with a small hole allowing only one structure to be illuminated on the chip, (c) a photo of the complete set-up.

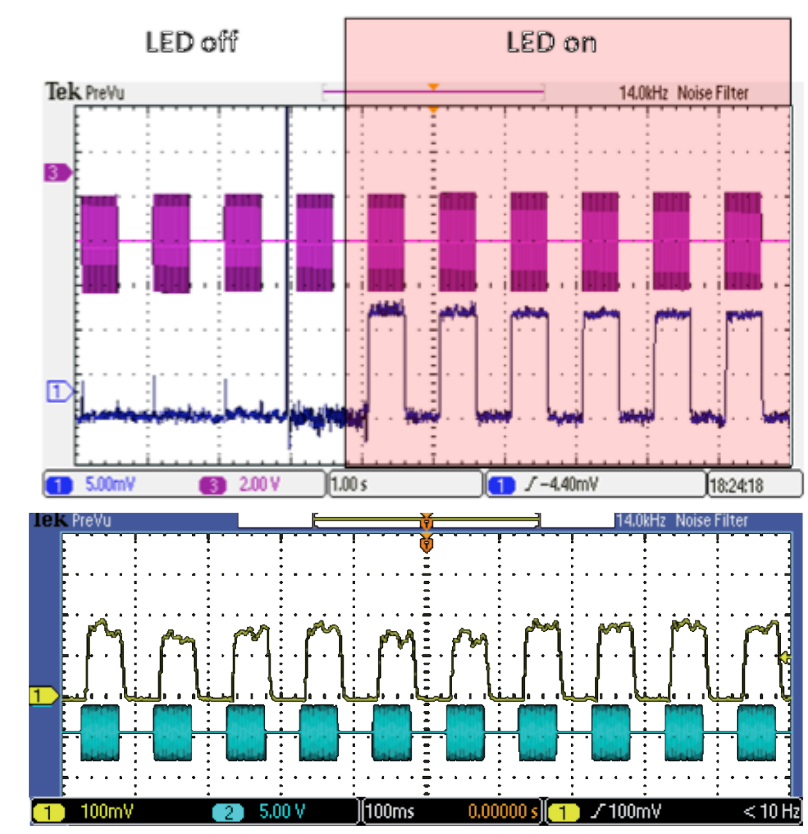

Fig. 5. Measured results. (top) Structure \#4: trace no. 1 is the output, no. 3 is the input modulation on the PN junctions. DC offset $V_{\mathrm{DC}}=-3.15 \mathrm{~V}, V_{\mathrm{pp}}=$ $4.4 \mathrm{~V}, f=1 \mathrm{kHz}, f_{\mathrm{env}}=1 \mathrm{~Hz}$. Lock-in to 2 nd harmonic, integration $3 \mathrm{~ms}$. (bot.) Struc. \#5: tr- 1 output, tr- 2 input, $V_{\mathrm{DC}}=-4.5 \mathrm{~V}, V_{\mathrm{pp}}=7 \mathrm{~V}, f_{\mathrm{env}}=10 \mathrm{~Hz}$, int.1ms. LED: $\lambda=1.55 \mu \mathrm{m}, I=90 \mathrm{~mA}, V=1.28 \mathrm{~V}$, optical output $\approx 4 \mathrm{~mW}$.

instrumentation and the speed of modulation is fundamentally limited by the junction capacitance.

\section{CONCLUSiON}

Here we have demonstrated a solution for the unresolved need for optical communication compatible with unmodified, mainstream silicon-based VLSI technology. Implementing this technology together with some standard solutions for power/data-in will enable CMOS chips to achieve both power and data transfer without any bond wires. Apart from the silicon photonics, this concept can also address packaging and encapsulation challenges faced in many lab-on-chip applications, for example a multichannel System on Chip for rapid point-of-care gene detection [18]. Demonstrated technique can readily achieve a relatively low bandwidth single communica- tion channel (single bitstream) sufficient for the lab-on-chip or similar applications. Speeds can be significantly improved by using narrow band-pass filters (instead of Lock-in amplifier) and by deploying several channels in parallel. Multi-channel applications have to address the question of cross-talk. In that case it would be possible to utilise the inherent advantage of this technology to be operational for different wavelengths $(1.3-8 \mu \mathrm{m})$ as well as various modulation techniques for the input voltage, with appropriate optical or electrical filtering at the output. The energy per bit is in the region of $1-10 \mathrm{~nJ} / \mathrm{bit}$ on the CMOS chip and the LED can consume up to $100 \mathrm{~mW}$, but this component is off chip. A very important aspect of our design is that it is fully implementable in any commerciallyavailable CMOS technology. Many techniques in the literature that are presented as CMOS-compatible refer to the fact that a CMOS process flow can be easily modified to accommodate the extra processing steps required. Our intention however, is to have no customisation whatsoever but to use unmodified digital IC technology. Given these aims and constraints (no control to doping levels, fabrication sequence, process steps, etc), we have successfully demonstrated a device structure and platform concept that achieves optical modulation.

\section{REFERENCES}

[1] R. Soref, "Mid-infrared photonics in silicon and germanium," Nature Photonics, vol. 4, no. 8, pp. 495-497, 2010.

[2] J. Di Liang, "Recent progress in lasers on silicon," Nature Photonics, vol. 4, no. 8, pp. 511-517, 2010.

[3] W. Ng, M. Lourenco, R. Gwilliam, S. Ledain, G. Shao, and K. Homewood, "An efficient room-temperature silicon-based light-emitting diode," Nature, vol. 410, no. 6825, pp. 192-194, 2001.

[4] L. Pavesi, L. Dal Negro, C. Mazzoleni, G. Franzo, and F. Priolo, "Optical gain in silicon nanocrystals," Nature, vol. 408, pp. 440-4, 2000.

[5] H. Rong, R. Jones, A. Liu, O. Cohen, D. Hak, A. Fang, and M. Paniccia, “A continuous-wave Raman silicon laser," Nature, vol. 433, no. 7027, pp. 725-728, 2005.

[6] G. Reed, G. Mashanovich, F. Gardes, and D. Thomson, "Silicon optical modulators," Nature Photonics, vol. 4, no. 8, pp. 518-526, 2010.

[7] C. Tang and G. Reed, "Highly efficient optical phase modulator in SOI waveguides," Electronics Letters, vol. 31, no. 6, pp. 451-452, 2002.

[8] A. Liu, R. Jones, L. Liao, D. Samara-Rubio, D. Rubin, O. Cohen, R. Nicolaescu, and M. Paniccia, "A high-speed silicon optical modulator based on a metal-oxide-semiconductor capacitor," Nature, vol. 427, no. 6975, pp. 615-618, 2004.

[9] Q. Xu, B. Schmidt, S. Pradhan, and M. Lipson, "Micrometre-scale silicon electro-optic modulator," Nature, vol. 435, pp. 325-327, 2005.

[10] M. Lipson, "Compact electro-optic modulators on a silicon chip," Sel. Top.Quantum Elec., IEEE Journal of, vol. 12, no. 6, pp. 1520-26, 2007.

[11] L. Liao, A. Liu, J. Basak, H. Nguyen, M. Paniccia, D. Rubin, Y. Chetrit, R. Cohen, and N. Izhaky, "40 Gbit/s silicon optical modulator for highspeed applications," Electronics Letters, vol. 43, no. 22, 2007.

[12] S. Preble, Q. Qianfan Xu, B. Schmidt, and M. Lipson, "Ultrafast alloptical modulation on a silicon chip," Optics Lett, vol. 30, no. 21, pp. 2891-93, 2005.

[13] G. Treyz, P. May, and J.-M. Halbout, "Silicon optical modulators at $1.3 \mu \mathrm{m}$ based on free-carrier absorption," IEEE Electron Device Lett., vol. 12 , no. 6, pp. 276-278, 1991.

[14] R. Soref and B. Bennett, "Electrooptical effects in silicon," IEEE Journal of Quantum Electronics, vol. 23, no. 1, pp. 123-129, 1987.

[15] R. Soref, "Silicon-based optoelectronics," Proceedings of the IEEE, vol. 81, no. 12, pp. 1687-1706, 2002.

[16] A. Serb, K. Nikolic, and T. Constandinou, "A CMOS-based light modulator for contactless data transfer: theory," Proc. SPIE Photonics West, vol. 7943, p. 794317, 2011.

[17] S. Sze and K. Ng, Physics of semiconductor devices. Wiley, 2007.

[18] D. Garner, H. Bai, P. Georgiou, T. Constandinou, S. Reed, L. Shepherd, W. Wong, K. Lim, and C. Toumazou, "A multichannel DNA SoC for rapid point-of-care gene detection,” Proc. IEEE ISSCC, pp. 492-3, 2010. 